\title{
Multidrug Resistance and Flaring up of Manifestation in Fistulizing Crohn's Disease after Surgery on a Perianal Lesion
}

\author{
Abeer S. Ali ${ }^{a}$ Omar S. Alhothali ${ }^{b}$ Abdulrahman A. Hammoudah ${ }^{b}$ \\ Abdullah Kh Alsaede ${ }^{b}$ Ayman A. Alraddadi ${ }^{b}$ \\ aPathology Department, Faculty of Medicine, Umm Al-Qurra University, Mecca, Saudi \\ Arabia; ${ }^{b}$ Fifth year medical student, Umm Al-Qurra University, Mecca, Saudi Arabia
}

Keywords

Crohn's disease $\cdot$ Fistula $\cdot$ Multidrug resistance

\begin{abstract}
Crohn's disease is a chronic inflammatory disease of the gastrointestinal tract with relapsing and remitting episodes. Abscesses and fistulas are the most common presentations of anorectal Crohn's disease. Antibiotics and surgical incision and drainage have been successful in treating perianal disease. We present here a 48-year-old woman with known case of Crohn's disease who presented with massive swelling in the perianal region with severe throbbing pain and high-grade fever, $38.2^{\circ} \mathrm{C}$; the surgeon noted a large perianal abscess near the anal verge with redness, hotness, and tenderness. One and a half months from perianal abscess surgery, culture sensitivity was done due to delayed wound healing and passage of greenish discharge, and it revealed highly resistant bacteria Proteus mirabilis, Escherichia coli, and Staphylococci. In conclusion, clinicians should be aware that abscess and fistula have a fair chance to develop in Crohn's disease patients who are using immunomodulating and immunosuppressant therapy. In abscess and fistula cases, surgery should be determined as soon as possible, and close clinical monitoring should be performed. We recommend routine screening for enteric fistula and culture sensitivity of any discharge prior to the initiation of any antibiotic. Appropriate intervention should then be undertaken.
\end{abstract}

\section{Karger $\stackrel{N}{\%}$}




\section{Introduction}

Crohn's disease (CD) is a chronic inflammatory disease of the gastrointestinal tract with relapsing and remitting episodes, and it can affect the entire gastrointestinal tract. Clinical presentation of the disease includes right lower quadrant abdominal pain, chronic diarrhea, weight loss, fatigue, and anorexia. In approximately $50 \%$ of CD patients, extraintestinal manifestation may occur, such as pauciarticular large joint arthritis and erythema nodosum [1].

Several perianal lesions including fissures, hemorrhoids, skin tags, fistulas and abscesses are associated with CD. These lesions can occur at any time in the course of the disease, and they occur in nearly half of the pediatric CD patients [2]. The most common presentations of anorectal CD are abscesses and fistulas. Twenty-six percent of patients will present with an abscess and an additional 29\% will present with a fistula [3]. Treatment of the perianal abscess is surgery, but we can avoid emergent surgery by antibiotic and percutaneous drainage [1].

About $66 \%$ of patients with inflammatory bowel disease (IBD) take corticosteroids, 14-20\% are treated with immunomodulator therapy, and 5\% use infliximab [4]. Around 15\% of patients with IBD are treated with antibiotics over a period of 12 years. Patients with CD experience frequent hospitalizations and the routine use of antibiotics during their course of the disease, which may predispose them to antimicrobial-resistant microorganisms [5]. According to one study [6], there was no difference between IBD patients and non-IBD patients in the risk of antibiotic resistance except for high antibiotic exposure. We report a case of antimicrobial-resistant organisms and flaring up of manifestation after perianal abscess surgery in a long-standing CD female patient.

\section{Case Presentation}

Here, we report a case of a 48-year-old woman who was diagnosed with CD at the age of 41. Prior to the diagnosis, there were multiple surgical excisions of perianal skin tags which were diagnosed falsely as external hemorrhoids. Since the age of 41, the patient's drug therapy consisted of immunosuppressive infliximab intravenous injections and immunosuppressive azathioprine and mesalamine, an aminosalicylate anti-inflammatory agent. This aggressive drug therapy was prescribed in 2013 due to the first 2 entero-enteric fistulas. After healing of the fistulas after 6 months which was assessed through MRI for entero-enteric fistulas imaging, the treatment with anti-TNF antibodies continued and installed till now. The patient condition was not stable; on the last 7 years after 2 years from diagnosis, increasing trash level of infliximab occurred, and the gastroenterologist stopped infliximab for 2 months, and he recommended continuing immunosuppressive azathioprine and mesalamine with prednisolone $50 \mathrm{mg}$ with gradual withdrawal of the dose and then subcutaneous adalimumab every 2 weeks. The condition was not improved; then, the patient was shifted to other monoclonal antibodies starting from certolizumab and vedolizumab and finally from December 2019 till now with ustekinumab (Stelara) and oral medications.

In December 2019, CT with contrast was performed as a routine follow-up, but the patient had severe anaphylactic reaction, and an aggressive course of oral corticosteroids was prescribed for 10 days. Missed diagnosis on reading the CT report. One week later after subsidence of anaphylactic reaction, a swelling in the perianal region with severe throbbing pain was felt and high-grade fever $38.2^{\circ} \mathrm{C}$; the surgeon noted a large perianal abscess near the anal verge with redness, hotness, and tenderness. A course of Augmentin $1 \mathrm{~g}$ was prescribed with analgesic and antipyretics for 10 days; 2 days later from diagnosis, an opened sinus tract occurred discharging large amount of pus. In January 2020, MRI fistula was done which noted

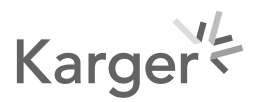


Fig. 1. MRI perianal fistula with small related horseshoe intersphincteric collection near the anal verge.

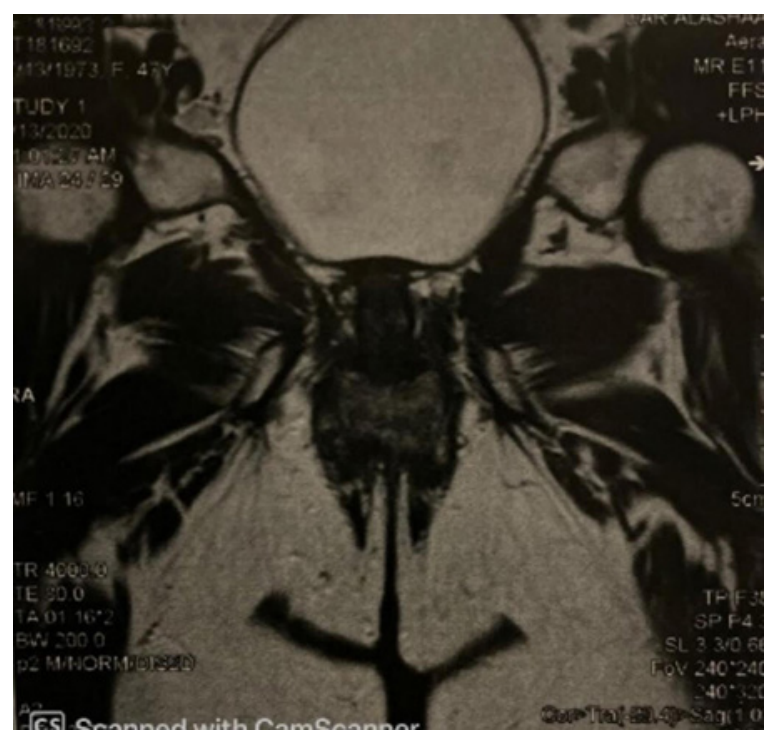

evidence of small right-sided intersphincteric horseshoe abscess $1.5 \times 1 \times 2.2 \mathrm{~cm}$ in intersphincteric space and extending from 7 to 9 o'clock continuous with right-sided low intersphincteric fistulous tract descending into the right side of the anal cleft. Fistulectomy operation was recommended, but due to unknown reason, the surgery was postponed till July 2020 where another MRI was done which was compared to previous imaging, and its opening was noted at 8 o'clock about $5.8 \mathrm{~cm}$ from the anal verge and descends at the intersphincteric space with small related horseshoe intersphincteric collection about $1.5 \mathrm{~cm}$ from the anal verge (shown in Fig. 1). Incision, excision of skin tags and fistulous tract which was sent to the pathologist, and drainage operation with antibiotics coverage were done, with recommendation to stop the immunomodulating therapy and immunosuppressant before and after the operation till complete healing and closure of the wound. Histopathology report revealed nonspecific inflammatory fistula showing granulation tissue lining consisting of newly formed small vascular channels, surrounded by fibroblastic proliferations with chronic inflammatory cellular infiltrations. The marginal surface epithelium shows acanthosis and hyperkeratosis with pseudoepitheliomatous hyperplasia (shown in Fig. 2a, b). One and a half months from operation, the surgeon prescribed culture sensitivity from the discharge, and it revealed highly resistant bacteria Proteus mirabilis and Escherichia coli (E. coli). Curam tablet (trimethoprim sulfamethoxazole) was prescribed for 14 days, but the condition was not improved. With the discharge passages, another culture sensitivity was done revealing P. mirabilis, E. coli, and Staphylococci. Antibiotic course was repeated with Flagyl $500 \mathrm{mg}$ tablet, the opening closed after 4 months, and the patient was diagnosed with CD; fecal calprotectin $2000 \mu \mathrm{g} / \mathrm{g}$, CRP $28 \mathrm{mg} / \mathrm{L}$ with abdominal distension, and passage of blood with defecation. The patient noted fecal incontinence, and the surgeon asked for MRI with contrast and examination of the fistulous tract under anesthesia to fulfill and evaluate the right diagnosis.

\section{Discussion}

We report a case of antimicrobial multidrug-resistant organisms and flaring up of manifestation after perianal abscess surgery in a patient with long-standing CD. Perianal abscesses are considered one of the most common surgical cases faced by both general and colorectal surgeons. Patients with perianal abscess present with severe pain, tenderness, swelling, and 

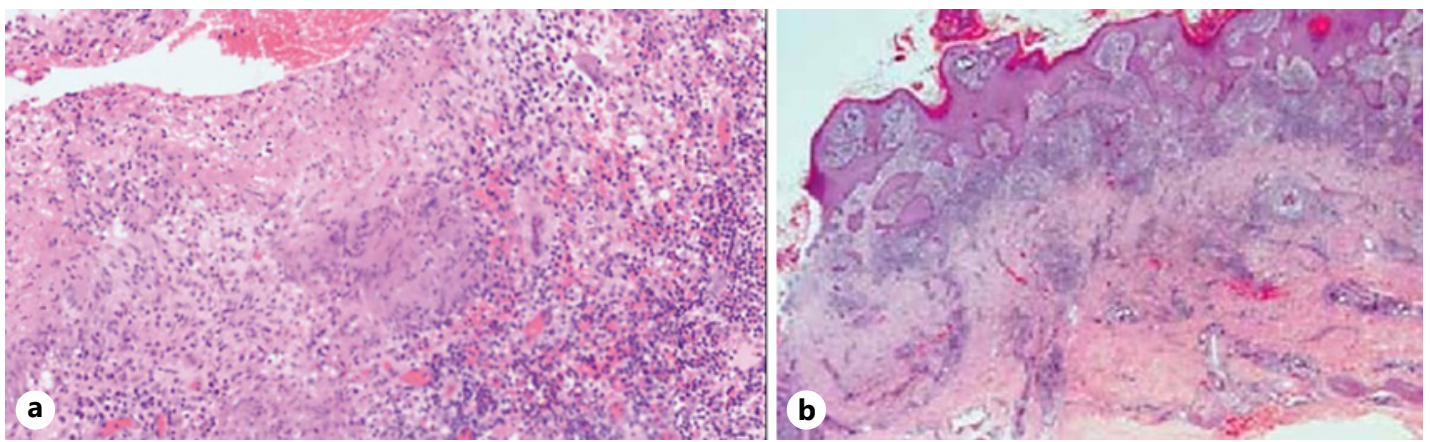

Fig. 2. a Nonspecific inflammatory fistula showing granulation tissue lining consisting of newly formed small vascular channels. b Acanthosis and hyperkeratosis with pseudoepitheliomatous hyperplasia. Power magnification for all microscopy images, $\times 10$ objective and $\times 10$ eyepiece.

fluctuation on rectal examination. Extension of a cryptoglandular infection or obstruction of a perianal fistula is the main cause of perianal abscesses [3]. The management of perianal abscesses includes treatment of systemic symptoms with broad-spectrum antibiotics, but the definitive management is surgical incision and drainage [7] which has been delayed in our case.

Antibiotics have been successful in treating perianal disease and have been used on the basis that the bacterial flora, especially anaerobes, contributes to perianal sepsis [7]. The routine use of antibiotics may be considered as malpractice and does not improve healing times in CD. Their use should be limited to treating patients with impaired resistance to infection such as immunosuppression, diabetes, and extensive cellulitis. A previous study showed resistance of Gram-negative bacteria to ciprofloxacin in CD patients with intraabdominal abscesses [8]. In our case, one and a half months after perianal abscess surgery, culture sensitivity was done and revealed highly resistant bacteria P. mirabilis and E. coli and Staphylococci.

Immunosuppressants such as corticosteroids and immunomodulators such as azathioprine are common therapies for patients with CD. Systemic corticosteroids have been widely used in CD remission induction. Immunomodulators are effective for remission maintenance, but not for remission induction [9]. They have not shown to be a risk factor for infectious complications following surgery. However, preoperative usage of CS is associated with postoperative infectious complications [10]. The presented case has been on multiple courses of CS which could be a cause of her development of postoperative wound infections.

The medical management of IBD has been revolutionized by TNF inhibitors, which include infliximab, adalimumab, golimumab, and certolizumab pegol. Anti-TNF agents inhibit disease activity and contribute to the healing of the mucosa. In the ACCENT II trial, which assessed infliximab as a maintenance therapy for fistulizing CD, 39\% of patients in the infliximab maintenance arm had complete closure of all drainage fistulas, compared with only $9 \%$ in the placebo arm [9]. Ustekinumab is another biologic therapy approved for the treatment of mild to serious CD patients. It is a monoclonal antibody that blocks the common p40 subunit of IL-12 and IL-23 [11]. Our patient stopped using infliximab because she had increasing trash level of the drug and was on ustekinumab, and she stopped using it before and after the surgery to improve healing because no enough data support its effect in fistula remission. In a recent systemic review and meta-analysis of 27 controlled trials, a moderatequality evidence was found to support the efficacy of ustekinumab to induce fistula remission, and more efficacy data are needed on this agent [11]. 
Fistulectomy is the excision of the complete fistula tract. It makes a larger wound compared to fistulotomy. This technique is usually used for intersphincteric or low transsphincteric perianal fistulae [12]. In our case, the patient had low intersphincteric fistulae and the surgeon decided to do fistulectomy, but the surgeon did not use Seton which can preserve the sphincter function, maybe because he anticipated that she will have fecal incontinence despite using Seton.

In conclusion, in CD patients who are using immunomodulating and immunosuppressant therapy, clinicians should be aware that abscess and fistula have a fair chance to develop. In cases in which abscess and fistula are suspected, surgery should be determined as soon as possible, and close clinical monitoring should be performed. We recommend routine screening for enteric fistula and culture sensitivity of any discharge prior to the initiation of any antibiotic. Appropriate intervention should then be undertaken.

\section{Statement of Ethics}

This case report was approved by the Biomedical Ethics Committee. Written informed consent was obtained from the patient for publication of this case report and any accompanying images.

\section{Conflict of Interest Statement}

The authors have no conflicts of interest to declare.

\section{Funding Sources}

There was no financial support.

\section{Author Contributions}

Abeer Ali: corresponding author, manuscript writing, and literature review. Omar Alhothali: manuscript writing and literature review. Abdulrahman Hammoudah: manuscript writing and literature review. Abdullah Alsaede: manuscript review and literature review. Ayman Alraddadi: literature review and manuscript writing. All authors read and approved the final manuscript.

\section{References}

1 Torres J, Mehandru S, Colombel J-F, Peyrin-Biroulet L. Crohn's disease. Lancet. 2017;389(10080):174155.

2 Griffiths AM. Specificities of inflammatory bowel disease in childhood. Best Pract Res Clin Gastroenterol. 2004; 18(3):509-23.

3 Lewis RT, Maron DJ. Efficacy and complications of surgery for Crohn's disease. Gastroenterol Hepatol. 2010; 6(9):587.

4 Hutfless SM, Weng X, Liu L, Allison J, Herrinton LJ. Mortality by medication use among patients with inflammatory bowel disease, 1996-2003. Gastroenterology. 2007;133(6):1779-86.

5 Epidemiological S. Epidemiological and clinical features of Spanish patients with crohn's disease. Spanish epidemiological and economic study group on crohn's disease. Eur J Gastroenterol Hepatol. 1999;11(10): 1121-7. 
6 Leung W, Malhi G, Willey BM, McGeer AJ, Borgundvaag B, Thanabalan R, et al. Prevalence and predictors of MRSA, ESBL, and VRE colonization in the ambulatory IBD population. J Crohns Colitis. 2012;6(7):743-9.

7 Singh B, Jewell D, George B. Perianal Crohn's disease. Br J Surg. 2004;91(7):801-14.

8 Park SK, Kim KJ, Lee SO, Yang DH, Jung KW, Ye BD, et al. Ciprofloxacin usage and bacterial resistance patterns in Crohn's disease patients with abscesses. J Clin Gastroenterol. 2014;48(8):703-7.

9 Gajendran M, Loganathan P, Catinella AP, Hashash JG. A comprehensive review and update on Crohn's disease. Dis Mon. 2018;64(2):20-57.

10 Aberra FN, Lewis JD, Hass D, Rombeau JL, Osborne B, Lichtenstein GR. Corticosteroids and immunomodulators: postoperative infectious complication risk in inflammatory bowel disease patients. Gastroenterology. 2003;125(2):320-7.

11 Lee MJ, Parker CE, Taylor SR, Guizzetti L, Feagan BG, Lobo AJ, et al. Efficacy of medical therapies for fistulizing Crohn's disease: systematic review and meta-analysis. Clin Gastroenterol Hepatol. 2018;16(12):1879-92.

12 Keshaw H, Foong KS, Forbes A, Day RM. Perianal fistulae in Crohn's disease: current and future approaches to treatment. Inflamm Bowel Dis. 2010;16(5):870-80. 